Benzol mehr bei $g$ zurückfliesst, Hahn $c$ geöffnet, wodurch das mit p-Phenylendiamin gesättigte Benzol in das aus dünnem Bleiblech gefertigte cylinderförmige Gefäss $O$ läuft. Ist das geschehen, so wird Hahn $c$ wieder geschlossen und das in dem Bottich $E$ befindliche Wasser, welches das Gefäss $C$ umgibt, durch Einleiten von Dampf erhitzt. Dadurch destillirt das Benzol allmäblich hoch, wird in dem Kühler $D$ condensirt und läuft in dünnem Strahl durch den Vorstoss $g$ nach $A$ zurück. Ist alles Benzol aus $C$ nach $A$ destillirt, so wird ev. unter Zusatz von etwas Benzol die Extraction in der vorher beschriebenen Weise begonnen und so oft wiederholt, bis alles $p$-Phenylendiamin ausgezogen ist. Dann wird das Gefäss $A$ von Neuem beschickt und wie bekannt das in $C$ befindliche Benzol mehrmals durchdestillirt. Dadurch wird das Gefäss $C$ allmählich mit reinem $\mathrm{p}$-Phenylendiamin gefüllt. Um dasselbe daraus zu entfernen, wird es zunächst durch Destillation rom Benzol befreit, welches dann bei $g$, ohne mit $A$ verbunden zu sein, in einer besonderen Vorlage aufgefangen wird. Nach dem Öffnen des weiten Mannlochs $h$, kann das rückständige $p$-Phenylendiamin mit Leichtigkeit aus $C$ entfernt werden.

Phenylenblau. Schmelze mit rohem p-Phenylendiamin. In einem eisernen Rührkessel werden $100 \mathrm{~g}$ p-Phenylendiaminbase und $35 \mathrm{~g}$ salzsaures $\mathrm{p}$-Phenylendiamin bei $150^{\circ}$ geschmolzen und in die geschmolzene Masse langsam $36 \mathrm{~g} \alpha$-Nitronaphtalin eingetragen. Es ist anzurathen, die Temperatur dabei nicht über $170^{\circ}$ steigen zu lassen, nach dem Eintragen aber etwa 3 Stunden auf 180 bis $190^{\circ}$ zu erhitzen. Eine Probe löst sich dann grünlichblau in conc. Schwefelsäure. Die Schmelze, deren Gewicht $150 \mathrm{~g}$ beträgt, wird gepulvert und in $4 l$ Wasser, welche $200 \mathrm{~g}$ Salzsäure von $20^{\circ}$ Bé. enthalten, gelöst; dabei bleibt kein Rückstand. Durch Zusatz von $5 l$ Kochsalzlösung wird das gebildete Phenylenblau gefällt, dessen Menge nach dem Abfiltriren, Pressen und Trocknen $150 \mathrm{~g}$ beträgt. Der Farbstoff weicht in keiner Weise von dem im Handel befindlichen Phenylenblau $a b$, woraus sich die Brauchbarkeit der rohen $\mathrm{p}$-Phenylendiaminbase für diesen $\mathrm{Z}_{\text {weck }}$ ergibt.

Das ablaufende Filtrat ist violett gefärbt; der darin gelöste Farbstoff stellt eine röthere Modification dar, die durch Sättigen mit Kochsalz oder durch Natronlauge gefällt werden kann, dessen Menge etwa 15 bis $18 \mathrm{~g}$ beträgt, aber auch $\alpha$-Naphtylamin, durch Reduction des Nitronaphtalins entstanden, enthält.

\section{Über die}

Darstellung von Kaliumplatinchlorür.

Von

Max Gröger.

Nach dem Verfahren von Thomsen ${ }^{1}$ ) wird Kaliumplatinchlorid mit Wasser zu einem Brei verrübrt, erwärmt und feuchtes Kupferchlorür in solcher Menge eingetragen, dass noch ein kleiner Theil des Kaliumplatinchlorides unverändert bleibt, hierauf filtrirt. Beim Erkalten des Filtrates krystallisirt Kaliumplatinchlorür aus, welches nach dem Abgiessen der Mutterlauge durch Waschen mit Weingeist von Kupferchlorid befreit und durch Umkrystallisiren gereinigt wird.

Die Kupferchlorürmenge, welche bei diesem Verfahren verbraucht wird, ist weit grösser, als die Umsetzungsgleichung

$$
\mathrm{K}_{2} \mathrm{Pt} \mathrm{Cl}_{6}+2 \mathrm{Cu} \mathrm{Cl}=\mathrm{K}_{2} \mathrm{Pt} \mathrm{Cl}_{4}+2 \mathrm{Cu} \mathrm{Cl}_{2}
$$

verlangt. Das Kupferchlorür muss im Überschuss verwendet werden, wenn nicht ein allzugrosser Theil des Kaliumplatinchlorides unverändert bleiben soll; da ausserdem ein Theil des Kupferchlorüres durch die Einwirkung der Luft in unlösliches basisches Kupferchlorid übergeht, so entsteht eine ziemliche Menge Niederschlag, welche bei der darauffolgenden Filtration einen nicht unbeträchtlichen Theil der concentrirten Kaliumplatinchlorürlösung zurückhält. Ein Auswaschen des Niederschlages ist nicht thunlich, weil - abgesehen davon, dass das Filtrat sehr leicht trüb durch das Filter läuft - beim Verdampfen der Waschwässer durch Einfluss des Kupferchlorides Rückbildung von Kaliumplatinchlorid stattfindet. Die Mutterlauge, welche nach dem Auskrystallisiren des Kaliumplatinchlorüres bleibt, enthält noch viel von diesem Salze gelöst, welches aus demselben Grunde daraus durch Verdampfung nicht mehr gewonnen werden kann. In Anbetracht dieser Umstände beträgt die Ausbeute an reinem Kaliumplatinchlorür nur etwa 50 Proc. der theoretischen.

Wegen der Umkehrbarkeit des Vorganges, welcher dem Thomsen'schen Verfahren zu Grunde liegt, wird durch das dabei sich bildende Kupferchlorid eine vollständige Umsetzung verbindert. Wenn man daher ein Mittel hätte, das Kupferchlorid in dem Maasse, wie es sich bildet, beständig aus der Lösung fortzuschaffen, ohne dass die übrigen in der Lösung befindlichen Stoffe davon angegriffen würden, so müsste eine vollständige Umsetzung gelingen. Ein

1) J. pr. Chemie [2] 15, S. 295. 
Reagens, welches diesen Anforderungen nahezu entspricht, ist das Zinkoxyd; es wirkt auf gelöstes Kupferchlorid augenblicklich, auf Kupferchlorür und Kaliumplatinchlorür in der Kälte nur äusserst langsam ein. (Aus einer kochenden Kaliumplatinchlorürlösung scheidet es einen grauschwarzen platinoxydulhaltigen Niederschlag aus.)

Schüttelt man in einem verschlossenen Fläschchen äquivalente Mengen von Kupferchlorür und Kaliumplatinchlorid andauernd mit Wasser, so bemerkt man die Bildung eiper dunkelgrünlichbraunen Lösung und eines Niederschlages, in welchem man neben Kupferchlorür noch deutlich eine grosse Menge der gelben Körnchen des Kaliumplatinchlorides wabrnimmt; setzt man aber nach und nach Zinkoxyd hinzu und schüttelt nach jedem Zusatz, so verschwinden die gelben Körnchen bald und es entsteht ein bläulichgrüner, feinpulveriger Niederschlag von basischem Kupferchlorid. Hat dieser sich abgesetzt, so erscheint die darüberstehende Lösung rubinroth. Verdampft man die filtrirte Lösung bis zur Krystallisation und lässt erkalten, so zeigt sich, dass neben den rothen Krystallnadeln des Kaliumplatinchlorüres doch noch eine kleine Menge der gelben Krystallkörner des Kaliumplatinchlorides sich ausscheidet. Da daraus ersichtlich, dass auch ein Theil des Kupferchlorüres durch das Zinkoxyd der Reaction entzogen wird, so wurde bei weiteren Versuchen die Menge des Kupferchlorüres allmählich bis auf das Doppelte erhöht. Nach der darauffolgenden Ausfällung durch Zinkoxyd gab das Filtrat beim Verdampfen bis zur Krystallisation allerdings nur mehr Krystalle von Kaliumplatinchlorür, allein der Niederschlag von basischem Kupferchlorid enthielt Platin beigemengt.

Die Mutterlauge von der ersten Krystallisation gibt noch eine zweite Krystallisation von Kaliumplatinchlorür. Die zweite Mutterlauge, die schon sehr reich an Zinkchlorid geworden, unterliegt bei weiterem Verdampfen einer Zersetzung, es entstehen bräunlichgelbe krystallinische Krusten und braune, fockige Ausscheidungen, die platinhaltig sind.

Da in dem Filtrat vom basischen Kupferchlorid neben Kaliumplatinchlorür und -chlorid nur noch Zinkchlorid zu vermuthen war, erstere in Alkohol unlöslich, letzteres darin sebr leicht löslich ist, so wurde versucht, die Platinsalze durch Ausfällung mit Alkohol abzuscheiden. Es fällt durch Zusatz von Alkohol zwar ein grosser Theil des Kaliumplatinchlorüres als rosenrothes Krystallmehl aus, allein das Filtrat davon ist Ch. 97 . nicht, wie man erwarten sollte, farblos, sondern stark gelb gefärbt und enthält neben Zinkchlorid eine beträchtliche Menge von Platinsalzen gelöst. Beim Verdampfen dieser alkoholischen Lösung auf dem Wasserbade scheidet sich Platinschwarz ab. Nach mehrfach abgeänderten Versuchsbedingungen bin ich schliesslich zu folgendem Verfahren gelangt.

In einem Fläschchen mit eingeschliffenem Glasstöpsel werden $8 \mathrm{~g}$ krystallisirtes Kupferchlorid $\left(\mathrm{Cu} \mathrm{Cl}_{2}+2 \mathrm{H}_{2} \mathrm{O}\right)$ in $100 \mathrm{~g}$ kalten Wassers gelöst und mit $1,4 \mathrm{~g}$ Zinkstaub kräftig geschüttelt. Ist ein gleichförmiger Niederschlag von Kupferchlorür entstanden, so fügt man $10 \mathrm{~g}$ feinzerriebenes Kaliumplatinchlorid hinzu und schüttelt wieder, dann gibt man $5 \mathrm{~g}$ Zinkoxyd in das Fläschchen und schüttelt, bis die gelben Körnchen des Kaliumplatinchlorides verschwunden sind. Nun filtrirt man den Niederschlag, der aus basischem Kupferchlorid und etwas überschüssigem Zinkoxyd besteht, ab und wäscht ihn mit kaltem Wasser aus. Filtrat und Waschwässer werden mit wenigen Tropfen Salzsäure angesäuert (um die Bildung basischen Zinkchlorides zu verhindern), auf dem Wasserbade bis zur eben eintretenden Bildung einer Krystallhaut verdampft und erkalten gelassen. Es scheidet sich Kaliumplatinchlorür in Krystallnadeln aus. Man bringt das Ganze auf einen Trichter, lässt die Mutterlauge abtropfen und verdampft sie auf dem Wasserbade neuerlich bis zur Bildung einer Krystallhaut, lässt erkalten und bringt die zweiten Krystalle auf denselben Trichter. Die abtropfende zweite Mutterlauge wird nicht weiter auf Kaliumplatinchlorür verarbeitet; man fällt daraus zweckmässig durch Zinkstaub Platinschwarz aus. Die auf dem Trichter befindlichen Krystalle werden zur Entfernung der anhaftenden Zinkchloridlösung vor der Saugpumpe zunächst mit etwas salzsäurehaltigem, dann mit reinem Alkohol ausgewaschen (es ist dazu nur wenig Alkohol erforderlich) und im Dunkeln bei gewöhnlicher Temperatur trocknen gelassen. Die Krystallmasse wird sodann mit dem fünffachen Gewicht kalten Wassers geschüttelt, Kaliumplatinchlorür geht dadurch vollständig in Lösung, das beigemengte Kaliumplatinchlorid bleibt ungelöst und wird durch Filtration entfernt; aus dem Filtrat krystallisirt das Kaliumplatinchlorür vollkommen rein und vollständig aus.

Nach diesem Verfahren erhält man aus $10 \mathrm{~g}$ Kaliumplatinchlorid 6 bis $6,5 \mathrm{~g}$ reines Kaliumplatinchlorür in schönen, rubinrothen Krystallen, d. h. 70 bis 76 Proc. der theoretischen Ausbeute. 
Um die Reinheit des Salzes festzustellen, wurde eine grössere Menge der concentrirten wässerigen Lösung auf kochendem Wasserbade durch Zusatz von reinem Alkohol reducirt. Die von dem Platin, welches als spiegelnder Beschlag an der Gefässwand sich abscheidet, abgegossene farblose Lösung blieb auf Zusatz von Sodalösung vollkommen klar, war also frei von Zink.

Zum Vergleiche wurden die von Carey Lea $a^{2}$ veröffentlichten Methoden zur Omwandlung des Kaliumplatinchlorides in -chlorür durch saures Kaliumsulfit in Bezug auf Ausbeute untersucht. Nach der einen Vorschrift Carey Lea's werden $12 \mathrm{~g}$ Kaliumplatinchlorid mit $9 \mathrm{~g}$ saurem Kaliumsulfit und $160 \mathrm{~g}$ Wasser im bedeckten Gefäss auf kochendem Wasserbade erwärmt, bis die Lösung rein roth geworden (wozu etwa 12 Stunden erforderlich sind), dann bis zur Krystallisation verdampft. Im Ganzen gaben drei Krystallisationen $8 \mathrm{~g}$ Krystalle. Die erste Krystallisation enthielt wenig, die zweite mehr, die dritte schon sebr viel saures Kaliumsulfat beigemengt; es war ein dreimaliges Umkrystallisiren aus Chlorkaliumlösung erforderlich, um die Krystalle sulfatfrei zu erhalten. Es wurden schliesslich $6,5 \mathrm{~g}$ reine Krystalle erhalten; ein zweiter ganz gleicher Versuch ergab $6,4 \mathrm{~g}, \mathrm{~d}$. $\mathrm{s}$. etwa 63 Proc. der theoretischen Ausbeute.

Nach der zweiten Vorschrift desselben Forschers wurden $12 \mathrm{~g}$ Kaliumplatinchlorid mit $6 \mathrm{~g}$ saurem Kaliumsulfit, 6 g Kaliumchlorid und $150 \mathrm{~g} \mathrm{Wasser}$ in einem Kolben 25 Minuten zum Kochen erhitzt, die Lösung filtrirt und krystallisiren gelassen. Es blieben 2 g Kaliumplatinchlorid auf dem Filter, das Filtrat gab bei der Krystallisation im Ganzen $7,5 \mathrm{~g}$ sulfathaltiger, nach dem Umkrystallisiren $6 \mathrm{~g}$ reiner Krystalle, d. s. 59 Proc. der theoretischen Ausbeute.

Die gleichfalls von Carey Lea beschriebene theilweise Entchlorung des Kaliumplatinchlorides durch unterphosphorigsaures $\mathrm{Ka}$ lium habe ich wegen der Schwierigkeit eine zu weitgehende Reduction zu vermeiden nicht angewendet, hingegen versucht, dieses Reductionsmittel durch das energisch reducirend wirkende unterschwefligsaure Kalium $\left(\mathrm{K} \mathrm{S} \mathrm{O}_{2}\right)$ zu ersetzen. Durch Schütteln einer Lösung von $10 \mathrm{~g}$ saurem Kaliumsulfit in $150 \mathrm{~g}$ Wasser mit $5 \mathrm{~g}$ Zinkstaub und darauffolgende Filtration wurde eine Lösung, die untersch wefligsaures Kalium enthielt, bereitet. $\mathrm{Zu} 10 \mathrm{~g}$ Kaliumplatinchlorid, welches in einem Kölbchen mit $50 \dot{g}$ Wasser zu lebhaftem Kochen erhitzt war, liess ich diese

2) Chem. Centralbl. 1895, I, 147.
Lösung aus einer Pipette rasch zutropfen bis zum Verschwinden des Kaliumplatinchloridniederschlages. Die rothe Lösung wurde bis zur Bildung einer Krystallhaut verdampft. Beim Erkalten krystallisirten 7,7 g Kaliumplatinchlorür aus. Aus der Mutterlauge krystallisirt hauptsächlich nur mehr Kaliumsulfat aus. Die Krystalle von Kaliumplatinchlorür mussten, um sie von anbaftendem Kaliumsulfat zu befreien, dreimal umkrystallisirt werden, so dass im Ganzen nur $5,5 \mathrm{~g}$ reines Kaliumplatinchlorür, d. s. 65 Proc. der theoretischen Ausbeute, erbalten wurden. Das unterschwefligsaure Kalium (wenigstens in Form der angewendeten Lösung) gewährt demnach dem sauren Kaliumsulfit gegenüber keinen wesentlichen Vortheil.

Setzt man, nachdem der Niederschlag des Kaliumplatinchlorides in der kochenden Flüssigkeit verschwunden, noch weiter obige Kaliumhyposulfitlösung $z u$, so wird die rothe Lösung rasch blasser, färbt sich dann gelblich und gibt bei grossem Überschuss dieses Reductionsmittels einen orangegelben flockigen Niederschlag. Der abfiltrirte, gewaschene und getrocknete Niederschlag gibt beim $\mathbf{E r}-$ hitzen Schwefeldioxyd ab und hinterlässt Platinschwarz; mit verdünnter Salzsäure gibt er eine dunkelbraune Lösung. - Eine genauere Untersuchung über die Zusammensetzung dieses Niederschlages ist im Gange.

Schliesslich wurde noch das von Pizzighelli und $H \ddot{H}$ ül ${ }^{3}$ ) empfohlene Verfahren zur Darstellung von Kaliumplatinchlorür aus Wasserstoffplatinchlorid in Vergleich gezogen. Darnach wird durch eine Lösung von $50 \mathrm{~g}$ krystallisirtem Wasserstoffplatinchlorid in $100 \mathrm{~g}$ Wasser auf kochendem Wasserbade nur so lange ein rascher Strom gewaschenen Schwefeldioxydes durchgeleitet, bis eine Probe der Flüssigkeit mit concentrirter Salmiaklösung eben keinen Niederschlag mehr gibt. $Z u$ der erkalteten Flüssigkeit setzt man dann unter Umrübren eine heisse Lösung von $25 \mathrm{~g}$ Kaliumchlorid in $50 \mathrm{~g}$ Wasser und bringt den nach 24 stündigem Stehen gebildeten Krystallbrei auf ein Filter, wäscht zunächst mit sehr wenig Wasser, dann mit Alkohol bis zum Verschwinden der sauren Reaction und lässt im Dunkeln trocknen. Es sollen nach dieser Darstellungsweise etwa 93 Proc. der nach der Theorie zu erwartenden Menge von $\mathrm{Ka}$ liumplatinchlorür erhalten werden, eine $\mathrm{An}^{-}$ gabe, die ich nicht bestätigen kann. In zwei genau nach obiger Vorschrift ausgeführten Versuchen erhielt ich aus je $50 \mathrm{~g}$

3) Encyklopädie der Photograph., Heft 15, 
reinem, trockenem, krystallisirtem Wasserstoffplatinchlorid (dessen der Formel $\mathrm{H}_{2} \mathrm{Pt} \mathrm{Cl}_{6}$ $+6 \mathrm{H}_{2} \mathrm{O}$ entsprechender Platingehalt von 37,6 Proc. vorher festgestellt worden war) $26,8 \mathrm{~g}$ bez. $27,1 \mathrm{~g}$ Kaliumplatinchlorür, d. s. 66,9 bez. 67,7 Proc. der theoretischen Ausbeute.

Auch die Angabe, dass das so erhaltene Krystallmehl völlig reines Kaliumplatinchlorür sei, ist nicht ganz zutreffend; es enthält eine kleine Menge saures Kaliumsulfat (durch Einwirkung des absichtlich im Überschuss zugesetzten Kaliumchlorides auf die in der reducirten Lösung enthaltene freie Schwefelsäure entstanden) und etwas Kaliumchlorid. Wollte man diese Beimengungen vollständig wegschaffen, so müsste man den auf dem Filter befindlichen Krystallbrei mit grösseren Mengen Wasser waschen, bevor man durch Alkohol die freie Säure wegwäscht, wodurch die Ausbeute wegen der Leichtlöslichkeit des Kaliumplatinchlorüres eine noch weitere Verminderung erführe. Für die $\mathrm{Z}$ wecke des Platindruckes sind diese geringen Beimengungen ohne Belang, ein Umkrystallisiren daher überflüssig; anders aber, wenn es sich um die Darstellung vollkommen reinen Kaliumplatinchlorürs handelt. Durch Umkrystallisiren des bei obigen Versuchen erhaltenen Krystallmehles wurden schliesslich nur 60 Proc. der theoretischen Ausbeute an reinem Kaliumplatinchlorür erhalten.

K. k. Staatsgewerbeschule in Wien I, Januar 1897.

Beiträge und Bemerkungen zur gerichtlichchemischen Weinanalyse.

Von

\section{Arthur Borntraeger.}

III. Zur Bestimmung des reducirenden Zuckers in Mosten, süssen und gewöhnlichen Weinen durch Titriren mit Fehling'scher Flüssigkeit.

Seit dem Jahre 1888 habe ich, namentlich in dieser Zeitschrift, ausgedehnte Angaben gemacht über die Bestimmung des Zuckers in Mosten und Weinen durch Titrirung. Jetzt möchte ich auf die Frage zurückkommen, indem ich meine Vorschläge und die wichtigsten Ergebnisse der früheren Untersuchungen kurz zusammenfasse und sie den auch neuerdings von Anderen gemachten Äusserungen gegenüberstelle. Mein Vorschlag ${ }^{1}$, den Zucker bei der Analyse von Mosten und Weinen allgemein auf Invert-

j) L'Orosi 1888, 325; Z. angew. Chem. 1889, 477, 538; 1892, 358: Z. anal. Chem. 1895, 19. zucker zu berechnen und nicht mehr auf Dextrose, wie dagegen die deutsche Verordnung rom Jahre 1884, die österreichische von 1886 und die italienische von 1889 vorgeschrieben hatten, ist jetzt wohl allgemein angenommen worden. Die Berechnung auf Invertzucker ist $u$. a. auch in der amtlichen "Anweisung zur chemischen Untersuchung des Weines" (d. Z. 1896, 498) vorgeschrieben, welche der Reichskanzler am 25. Juni dieses Jahres kundgégeben hat.

Für die Ausführung der Titrirungen empfehle ich die von mir angegebene Arbeitsweise $\left.^{2}\right)$.

Das von mir benutzte Verfahren zur Vorbereitung der Moste und Weine für die Titrirungen ist eine leichte Modificirung der früher $^{3}$ ) von mir vorgeschlagenen Methode und besteht nunmehr in Folgendem ${ }^{4}$ ).

a) Weine. Man neutralisirt ein bestimmtes Weinvolum in der Kälte annähernd und schliesslich in der Wärme genau mit Alkalilauge. Stark kohlensäurehaltige Weine sind zuvor kräftig durchzuschütteln, um die Hauptmasse der Kohlensäure zu vertreiben. Die neutrale Flüssigkeit wird auf dem Wasserbade zu etwa der Hälfte ihres Volums eingeengt. Wenn Weissweine vorliegen, so setzt man nach dem Erkalten $1 / 20$ rom Weinvolum an Bleiessig $\mathrm{Ph}$. G. III hinzu, bei Rothweinen dagegen 1/10 Volum. Sofern es sich um Weine mit weniger als 1 Proc. Zucker handeln sollte, fügt man nach 10 Minuten der nicht filtrirten Flüssigkeit $1 / 20$ bis $1 / 10$ Vol. einer kaltgesättigten Auflösung von Glaubersalz hinzu, füllt zum ursprünglichen Weinvolum auf, filtrirt nach weiteren 10 Minuten durch ein nicht benässtes Filter, ohne nachzuwaschen, und titrirt das Filtrat nach eventueller Verdünnung desselben.

b) Moste. Diese können in gleicher Weise verarbeitet werden, wie die Weine mit mehr als 1 Proc. Zucker.

Bei Süssweinen und Mosten kann auch die Wiederherstellung des ursprünglichen Weinvolums nach der Behandlung mit Bleiessig fortfallen, indem man das Ganze direct in der für die Titrirungen erforderlichen Weise verdünnt und nun erst filtrirt. Es könnte bei diesen Flüssigkeiten auch die ganze Vorbehandlung - Neutralisation, Eindampfen und Fällen mit Bleiessig - unterbleiben, sofern nicht rothe Süssweine vorliegen sollten. Bei Mosten und Süssweinen

2) Z. anal. Chem. 1895, 19.

3) L'Orosi 1888, 325; Z. angew. Chem. 1889, 477,$538 ; 1891,340,599$.

4) D. Z. 1894, 236, 454, 521, 554, 583; $1895,103$. 